\title{
ESTUDO BIOQUÍMICO DO POTENCIAL DA MEMBRANA AMNIÓTICA NA FIBROSE HEPÁTICA INDUZIDA EM RATOS
}

\section{Shaine Silva Maciel ${ }^{1}$ \\ Luciana Barros Sant'Anna²}

Resumo: A fibrose hepática causada por obstrução do ducto biliar induz alterações tanto na estrutura como na função do fígado, e pode levar a cirrose e insuficiência hepática, quando não tradada. A membrana amniótica humana (MA) devido às suas propriedades anti-inflamatórias e anti-fibróticas pode ser uma terapia alternativa. A proposta desse trabalho é analisar as alterações nos níveis séricos das principais enzimas celulares e da albumina, na fibrose hepática induzida pela ligadura do ducto biliar (LDB) e após o tratamento com a MA. Dez ratos foram divididos nos grupos $\angle D B$ e $L D B+M A$, e eutanasiados após 9 semanas da $\angle D B$. As amostras de sangue foram processadas bioquimicamente para análise da albumina $(A L B)$, fosfatase alcalina (FA) e transaminases (TGP e TGO). Comparando os grupos experimentais verificou-se que no grupo $\angle D B+M A$ houve a diminuição da $A L B$ e TGP e no grupo $L D B$ houve a diminuição da FA e TGO, entretanto sem apresentar diferença significante entre os grupos. Conclui-se que a MA, quando aplicada ao fígado no mesmo momento da indução da fibrose, parece não ter exercido efeito significativo na função hepática.

Palavras-chave: Bioquímica; Colestase; Fibrose Hepática; Membrana Amniótica.

\footnotetext{
${ }^{1}$ Nutríção/ Universidade do Vale do Paraíba, Brasil. E-mail: shainemaciel@gmail.com.

2 Instituto de Pesquisa e Desenvolvimento/Universidade do Vale do Paraíba, Brasil. E-mail: lucianabsa@gmail.com.
} 